\title{
Spinal Cord Injury Induces Serotonin Supersensitivity without Increasing Intrinsic Excitability of Mouse V2a Interneurons
}

\author{
Andreas Husch, Gabrielle N. Van Patten, Diana N. Hong, Moira M. Scaperotti, Nathan Cramer, \\ and Ronald M. Harris-Warrick \\ Department of Neurobiology and Behavior, Seeley Mudd Hall, Cornell University, Ithaca, New York, 14853
}

Denervation-induced plastic changes impair locomotor recovery after spinal cord injury (SCI). Spinal motoneurons become hyperexcitable after SCI, but the plastic responses of locomotor network interneurons (INs) after SCI have not been studied. Using an adult mouse SCI model, we analyzed the effects of complete spinal cord lesions on the intrinsic electrophysiological properties, excitability, and neuromodulatory responses to serotonin (5-HT) in mouse lumbar V2a spinal INs, which help regulate left-right alternation during locomotion. Four weeks after SCI, V2a INs showed almost no changes in baseline excitability or action potential properties; the only parameter that changed was a reduced input resistance. However, V2a INs became 100- to 1000-fold more sensitive to 5-HT. Immunocytochemical analysis showed that SCI caused a coordinated loss of serotonergic fibers and the 5-HT transporter (SERT). Blocking the SERT with citalopram in intact mice did not increase 5-HT sensitivity to the level seen after SCI. SCI also evoked an increase in 5- $\mathrm{HT}_{2 \mathrm{C}}$ receptor cluster number and intensity, suggesting that several plastic changes cooperate in increasing 5-HT sensitivity. Our results suggest that different components of the spinal neuronal network responsible for coordinating locomotion are differentially affected by SCI, and highlight the importance of understanding these changes when considering therapies targeted at functional recovery.

\section{Introduction}

Spinal cord injury (SCI) causes two major problems for the restoration of locomotor function (Rossignol, 2000). First, it eliminates both rapid synaptic and slow modulatory descending inputs from the brain that are essential for normal activation of the spinal locomotor central pattern generator (CPG). Second, spinal networks below the lesion may undergo slow denervationinduced plastic changes, including alterations in synaptic strength and intrinsic properties of spinal interneurons (INs), which could affect the proper function of the CPG. If the CPG networks become dysfunctional over time due to plastic changes resulting from the loss of descending inputs, simple restoration of these inputs may be inadequate to restore normal locomotor function. Post-SCI treadmill exercise, which drives sensory inputs to help retain normal CPG function, is one of the most

Received June 19, 2012; revised July 20, 2012; accepted July 25, 2012.

Author contributions: A.H., G.N.V.P., N.C., and R.M.H.-W. designed research;A.H., G.N.V.P., D.N.H., M.M.S., and N.C. performed research; A.H., G.N.V.P., D.N.H., M.M.S., N.C., and R.M.H.-W. analyzed data; A.H., G.N.V.P., D.N.H., M.M.S., N.C., and R.M.H.-W. wrote the paper.

This work was supported by grants from the National Institutes of Health (NS017323 and NS057599 to R.M.H.W.) and a fellowship by the German Science Foundation (DFG; Grant HU 1963/1-1 to A.H.). We thank F. Veermylen of the Cornell Statistical Consulting Unit and S. Dietz for assistance with statistical analysis of the data and K. Sharma at the University of Chicago for kindly providing the Chx10::eCFP mouse line. We thank J. Hardwick and B. Johnson for valuable comments on earlier versions of the manuscript and V. Patel, C. Benton, and B. McLaughon for technical assistance.

The authors declare no competing financial interests.

Correspondence should be addressed to Ronald M. Harris-Warrick, Department of Neurobiology and Behavior, Seeley Mudd Hall, Cornell University, Ithaca NY 14853. E-mail: rmh4@cornell.edu.

DOI:10.1523/JNEUROSCI.2995-12.2012

Copyright $\odot 2012$ the authors $\quad 0270-6474 / 12 / 3213145-10 \$ 15.00 / 0$ promising strategies to enhance functional motor output after SCI (Edgerton et al., 2004). However, the changes in IN properties that occur within the locomotor CPG after SCI, and their consequences for recovery, remain unknown.

SCI lesions the serotonergic fibers projecting from the brainstem to the spinal cord, significantly reducing the serotonin concentration caudal to the lesion (Carlsson et al., 1963; Hadjiconstantinou et al., 1984). As a consequence, the locomotor networks develop denervation supersensitivity to 5-HT (Shibuya and Anderson, 1968; Barbeau and Bédard, 1981). Reversing this loss of 5-HT can contribute to recovery, as serotonergic replacement strategies promote recovery of locomotion in rats (Ribotta et al., 2000; Antri et al., 2003, 2005; van den Brand et al., 2012). In terms of SCI-induced cellular changes, spinal cord motoneurons (MNs) have been most extensively studied. Rat sacral MNs increase their excitability 1 month after SCI, with a depolarized membrane potential, a hyperpolarized action potential (AP) threshold, and a significant increase in resting membrane resistance (Harvey et al., 2006a). Most importantly, these MNs show significantly enhanced bistability and generate plateau potentials driven by increases in persistent inward currents (PICs; Bennett et al., 2001b; Harvey et al., 2006b; Anelli et al., 2007). Chronic SCI also decreases RNA editing of the serotonin 5- $\mathrm{HT}_{2 \mathrm{C}}$ receptor in MNs (Murray et al., 2010), increasing their constitutive activity (Berg et al., 2001). These plastic changes of MN properties contribute to hyper-reflexia and spasticity, which are common complications after SCI (Elbasiouny et al., 2010; Murray et al., 2010).

Since SCI induces plastic changes in MN properties, we hypothesized that SCI would also induce changes in the INs of the 
locomotor CPG. Such changes would further reduce the capacity for functional recovery following SCI. Recently, we developed methods to study the firing properties and neuromodulation of identified INs in slices from the adult, behaviorally mature mouse spinal cord (Husch et al., 2011). As a consequence, we are now able to study how SCI affects the properties of these se IN without the confounds of postnatal developmental changes. Here, we address the effects of SCI on the firing properties and sensitivity to 5-HT of adult V2a INs. V2a INs are components of the locomotor CPG; they contribute to the control of left-right coordination, especially at high locomotor frequencies (Crone et al., 2008; Zhong et al., 2011), and their intrinsic electrophysiological properties have been very well studied (Dougherty and Kiehn, 2010; Zhong et al., 2010). We show that, in contrast to rat sacral MNs, SCI does not markedly alter V2a neuron firing properties, but significantly increases their sensitivity to serotonin.

\section{Materials and Methods}

Transgenic mice. Experiments were performed using Chx10::eCFP mice generated by Drs. Steven Crone and Kamal Sharma at the University of Chicago (Crone et al., 2008). The animal experimentation protocol was approved by the Institutional Animal Care and Use Committee at Cornell University and was in accordance with National Institutes of Health (NIH) guidelines.

SCI surgery. For the SCI surgery, 3- to 4-weekold mice (males and females) were anesthetized with intraperitoneal ketamine/xylazine $(100 \mathrm{mg} / \mathrm{kg}$ ketamine, $5 \mathrm{mg} / \mathrm{kg}$ xylazine). Buprenorphine (0.06 $\mathrm{mg} / \mathrm{kg}$ ) was administered subcutaneously. The dorsal skin was shaved and sterilized with 70\% ethanol and betadine. Using aseptic techniques, an incision was made dorsally above the thoracic region of the spinal cord to expose the vertebral column. A gap was made with forceps between the T8 and T9 vertebrae, and small scissors and fine forceps were used to completely transect the spinal cord transversely (Lapointe and Guertin, 2008). The incision was closed with tissue glue, and ketoprofen $(0.02 \mathrm{mg} / 10 \mathrm{~g} \mathrm{BW})$ and lactated Ringer's solution $(0.25 \mathrm{cc} / 10 \mathrm{~g} \mathrm{BW})$ were administered subcutaneously. As a sham control for SCI surgery, the same drug administration and surgical procedure described above were performed, but the spinal cord was not transected (referred to as intact mice). Injured mice received buprenorphine intraperitoneally twice daily for $2 \mathrm{~d}$ after surgery and ketoprofen intraperitoneally once daily for $4 \mathrm{~d}$ after surgery. Bladders were manually emptied as needed until bladder function returned. All mice were killed and used for electrophysiology or immunocytochemistry $4-5$ weeks after SCI or sham surgery.

Spinal cord slice procedure for electrophysiology. Coronal slices (250 $\mu \mathrm{m})$ were prepared from the lumbar spinal cord of adult mice (P43P70), as described previously (Husch et al., 2011). The lumbar spinal cord was removed from the dorsal surface of deeply anesthetized animals (150 mg/kg ketamine; $15 \mathrm{mg} / \mathrm{kg}$ xylazine) and sliced in ice-cold oxygenated $\left(95 \% \mathrm{O}_{2}\right.$ and $\left.5 \% \mathrm{CO}_{2}\right)$, glycerol-based artificial CSF (GACSF), which contained the following (in $\mathrm{mm}$ ): 222 glycerol, $3.08 \mathrm{KCl}, 1.18$ $\mathrm{KH}_{2} \mathrm{PO}_{4}, 1.25 \mathrm{MgSO}_{4}, 2.52 \mathrm{CaCl}_{2}, 25 \mathrm{NaHCO}_{3}$, and 11 D-glucose. Slices were transferred to $35^{\circ} \mathrm{C}$ oxygenated ACSF to recover for $45 \mathrm{~min}$. ACSF contained the following (in mM): $111 \mathrm{NaCl}, 3.08 \mathrm{KCl}, 1.18 \mathrm{KH}_{2} \mathrm{PO}_{4}, 1.25$ $\mathrm{MgSO}_{4} 2.52 \mathrm{CaCl} 2,25 \mathrm{NaHCO}$, and 11 D-glucose. Afterward, the slices passively cooled down to room temperature for at least $1 \mathrm{~h}$. CHx10::eCFP labeled V2a INs were visualized with a fixed-stage upright microscope (BX51WI, Olympus) using a $60 \times$ water-immersion objective (LUMPLFLN $60 \times \mathrm{W}$; Olympus) with infrared-differential interference contrast and fluorescence optics.

Perforated patch recordings. Slices were continuously superfused with oxygenated ACSF at a flow rate of $\sim 2 \mathrm{ml} / \mathrm{min}$. Neurons were isolated from most fast synaptic inputs with a combination of DL-2-amino-5phosphonopentanoic acid (AP-5, $10 \mu \mathrm{M})$ and 6-cyano-7-nitroquinoxaline2,3,-dione disodium salt hydrate $(10 \mu \mathrm{M})$ to block glutamatergic synapses, picrotoxin $(10 \mu \mathrm{M})$ to block GABAergic synapses, and strychnine $(10 \mu \mathrm{M})$ to block glycinergic synapses. All chemicals were obtained from Sigma-Aldrich. Amphotericin B perforated patch recordings (PPRs) were made as described in detail by Husch et al. (2011). The tip of the pipette was filled with intra- 
A

Intact
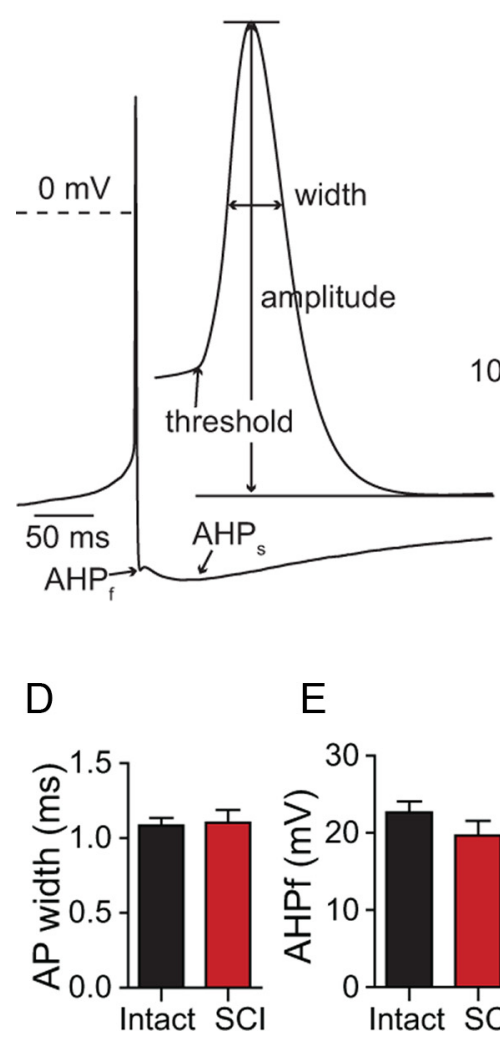

$\mathrm{E}$

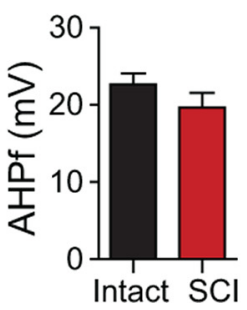

$\mathrm{SCl}$

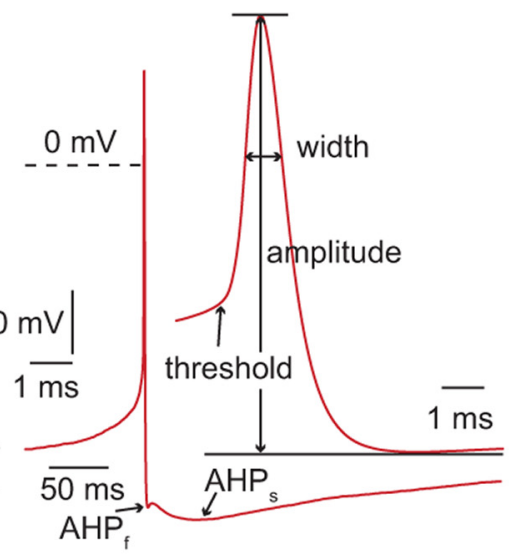

$\mathrm{F}$

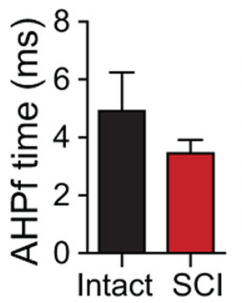

G

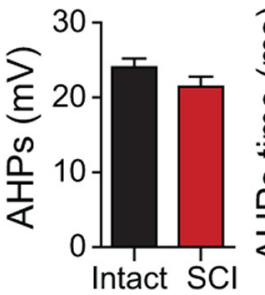

$\mathrm{B}$

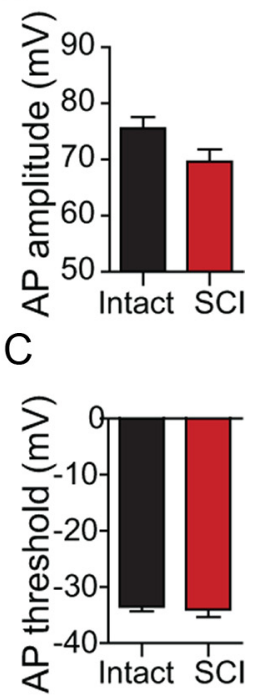

$\mathrm{H}$

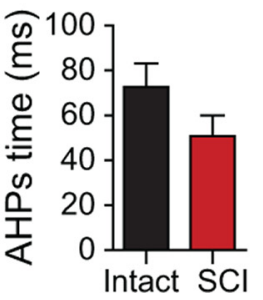

Figure 2. AP properties of intact and SCI V2a INs. A, Typical APs (signal-averaged by aligning peaks from $10-12$ APs) from intact and SCI cells at two different time scales illustrating the waveform parameters analyzed. $\boldsymbol{B}-\boldsymbol{H}$, Comparisons of AP amplitude $(\boldsymbol{B}), A$ P threshold $(\boldsymbol{C}), A$ P half-width $(\boldsymbol{D}), \operatorname{AHPf}(\boldsymbol{E})$, and its time course $(\boldsymbol{F})$, and slow sustained AHPs amplitude $(\boldsymbol{G})$ and time course $(\boldsymbol{H})$. Data in $\boldsymbol{B}-\boldsymbol{H}$ are means from 20 independent experiments in each group. Bars represent the mean. Error bars indicate SEM.

cellular solution containing the following (in mM) $135 \mathrm{~K}$-gluconate, $10 \mathrm{KCl}$, 10 HEPES, 0.1 EGTA, and $2 \mathrm{MgCl}_{2}$ (adjusted to $\mathrm{pH} 7.2$ with $\mathrm{KOH}, \sim 270$ $\mathrm{mOsm}$ ), and the pipette was backfilled with the same intracellular solution containing $1.2 \mathrm{mg} / \mathrm{ml}$ Amphotericin B and $1 \mathrm{mg} / \mathrm{ml}$ pluronic acid F127. Current-clamp recordings were obtained with a Multiclamp 700B amplifier (Molecular Devices) controlled by Clampex (pClamp 9; Molecular Devices). The junction potential was not corrected. Data were sampled at $10 \mathrm{kHz}(20$ $\mathrm{kHz}$ for AP shape analysis) and lowpass filtered at $2 \mathrm{kHz}$.

Electrophysiological data analysis. Data analysis was performed with Clampfit (Molecular Devices), Spike2 (CED), Igor Pro 6 (Wavemetrics), Graph Pad Prism (GraphPad Software), and MATLAB (MathWorks). For AP analysis, the membrane potential was adjusted with tonic current injection to set the firing frequency at $1 \mathrm{~Hz}$ or lower to elicit temporally isolated APs. The voltage threshold for AP generation was measured as the peak of the second derivative of voltage with time during the rising phase of the AP. The spike amplitude was measured from the peak of the AP to the peak afterhyperpolarization. The AP half-width was established at the voltage halfway from the spike threshold to the peak of the AP. To measure the membrane input resistance and rheobase (minimal current step for spike generation), neurons were held at $-60 \mathrm{mV}$ with holding current $\left(I_{\text {hold }}\right)$. Input resistance was estimated by averaging the response to small hyperpolarizing current pulses. To measure the spontaneous firing rate, the mean firing rate of $1210 \mathrm{~s}$ bins was averaged.

Immunocytochemistry and confocal microscopy. Intact and SCI mice were killed 4 weeks after SCI surgery. Mice were anesthetized with ketamine/xylazine intraperitoneally (150 mg/kg ketamine; $15 \mathrm{mg} / \mathrm{kg}$ xylazine), and perfused transcardially with perfusion buffer for $3 \mathrm{~min}$, and then $4 \%$ paraformaldehyde (PFA) in $0.1 \mathrm{~m}$ phosphate buffer for $20 \mathrm{~min}$. The spinal cord was removed, and the rostral portion of the upper lumbar enlargement postfixed in 4\% PFA, $0.1 \mathrm{~m}$ phosphate buffer solution for $1.5 \mathrm{~h}$ at $4^{\circ} \mathrm{C}$, then transferred to $30 \%$ sucrose solution for $12 \mathrm{~h}$ at $4^{\circ} \mathrm{C}$.
Coronal sections of the lesion site were also made to ensure completeness of the lesion. A cryostat was used to make $15 \mu \mathrm{m}$ transverse sections of the upper lumbar enlargement of an intact and an SCI cord, all of the same age and surgery date, placed on a single slide (Thermo Scientific SuperFrost Plus), with multiple pairs of slices on each slide. Slides were stored at $-20^{\circ} \mathrm{C}$ for up to 1 week before immunohistochemistry. A waterproof pen was used to enclose each group of slices on the slide. At room temperature, slides were rinsed in a PBS bath for $5 \mathrm{~min}$. Slides were incubated in $0.2 \%$ Triton $\mathrm{X}-100$ for $10 \mathrm{~min}$, then rinsed with three changes of PBS over $15 \mathrm{~min}$. Slices were incubated in peroxidase quenching buffer for $60 \mathrm{~min}$ at room temperature, and then $1 \%$ blocking reagent (Invitrogen Tyramide Signal Amplification Kit) for $60 \mathrm{~min}$ at room temperature. Each group of slices was incubated with one primary antibody (5- $\mathrm{HT}_{2 \mathrm{C}}$ 1:200, Abcam; SERT 1:1000, Immunostar; 5-HT 1:5000, Sigma), with one position incubated in $1 \%$ blocking reagent (no-primary antibody control), for $16 \mathrm{~h}$ at $4^{\circ} \mathrm{C}$. Slides were rinsed in $4^{\circ} \mathrm{C}$ PBS for 15 min, then incubated in secondary antibody conjugated horseradish peroxidase (Invitrogen TSA kit) diluted 1:100 in 1\% blocking solution for 45 $\mathrm{min}$ at room temperature, and rinsed in PBS for $15 \mathrm{~min}$. Slides were then incubated in Alexa Fluor 647 tyramide, diluted 1:100 in amplification buffer $/ 0.0015 \% \mathrm{H}_{2} \mathrm{O}_{2}$, for 16 min before final rinsing in PBS for $15 \mathrm{~min}$, and mounted with Fluor-Gel. A Leica TCS SP2 confocal microscope was used for imaging. A $20 \times$ objective lens was used to view tissue, and boxed regions (8-X zoom) of medial lamina VIII were imaged for analysis (see Fig. 5) using the $633 \mathrm{~nm}$ laser line. $Z$-series were taken through the $15 \mu \mathrm{m}$ tissue in $1 \mu \mathrm{m}$ steps.

Immunocytochemistry analysis. Images were analyzed using ImageJ (v.1.44p; NIH) and MATLAB. The images were deconvolved, and the mean intensity of the deconvolved no-primary controls for each mouse was subtracted from the images (Hutcheon et al., 2000). For 5-HT and SERT analysis, the percentages of pixels in each image above an auto- 
matic threshold were recorded, as well as the mean intensity of these pixels, and compared using the same threshold value for intact and SCI mice. Raw data were compared between slices of the same group on a slide, while ratios were used for between-experiment comparisons. For $5-\mathrm{HT}_{2 \mathrm{C}}$ receptor analysis, the number of receptor clusters was determined by first using the automatic threshold function in ImageJ. The same threshold was applied to SCI images from the same slide. The size and intensity of receptor clusters was determined by a set of MATLAB algorithms written in house. The program essentially finds the maxima of clusters, determines their boundaries using a threshold, and counts the pixels and total intensity value contained in each cluster. The MATLAB routines are available on request.

Statistical analysis. Descriptive and comparative statistics were performed using GraphPad Prism (GraphPad Software) or JMP (JMP software; SAS). Differences in parameters of two group means were determined with unpaired or paired $t$ tests as appropriate. For comparison of multiple treatments of individual cells, repeatedmeasures ANOVA was applied. To compare $f / I$ relations a regression analysis with a random coefficient model to correct for multiple measurements from each cell was performed. For statistical analysis of the immunocytochemistry data, the multilevel model (also known as mixed effect model) was used. In this statistical model, the intensity data and area data were the response variable, the mouse pair and mouse within each pair were set as the random effect, and the type of mouse (SCI or intact mouse) was set as the fixed effect. A significance level of 0.05 was accepted for all tests. Data are given as mean \pm SE.

\section{Results}

V2a INs do not show increased excitability following SCI

Chx10::eCFP mice, which express eCFP in spinal V2a INs (Zhong et al., 2010), were used in these studies. Complete spinal transections at T8-T9 were performed at 4 weeks of age, when adult locomotion has stabilized. Four weeks after SCI, we made long-term PPRs in eCFP-expressing spinal V2a INs (Fig. 1A). In marked contrast to the hyperexcitability of rat sacral MNs after SCI (Harvey et al., 2006b), we did not find a major increase in baseline excitability in mouse V2a INs 1 month after SCI. After blocking fast excitatory and inhibitory synaptic input with a combination of glutamate, GABA, and glycine antagonists, similar fractions of V2a neurons were silent or spontaneously active in intact and SCI populations (Fig. $1 B$; intact: $72 \%$ active, $21 / 29$; SCI: 50\%, 10/20; $p=$ $0.1 ; \chi^{2}$ test of independence). Mean AP frequencies in the active intact and SCI

\section{A Intact}

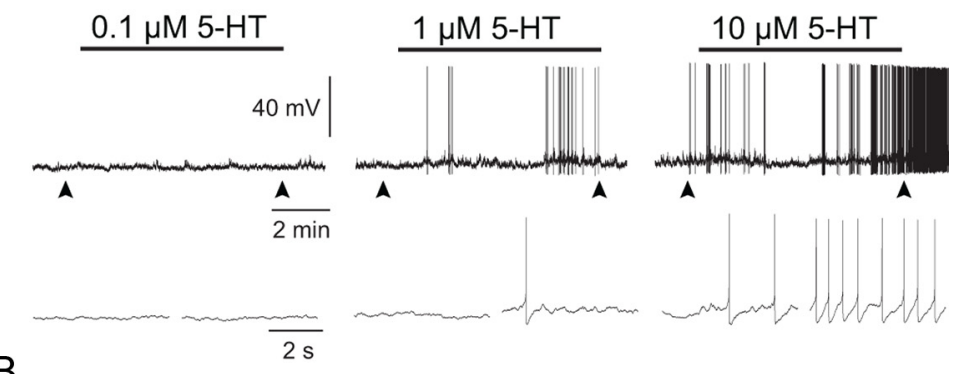

B

\section{$\mathrm{SCl}$}
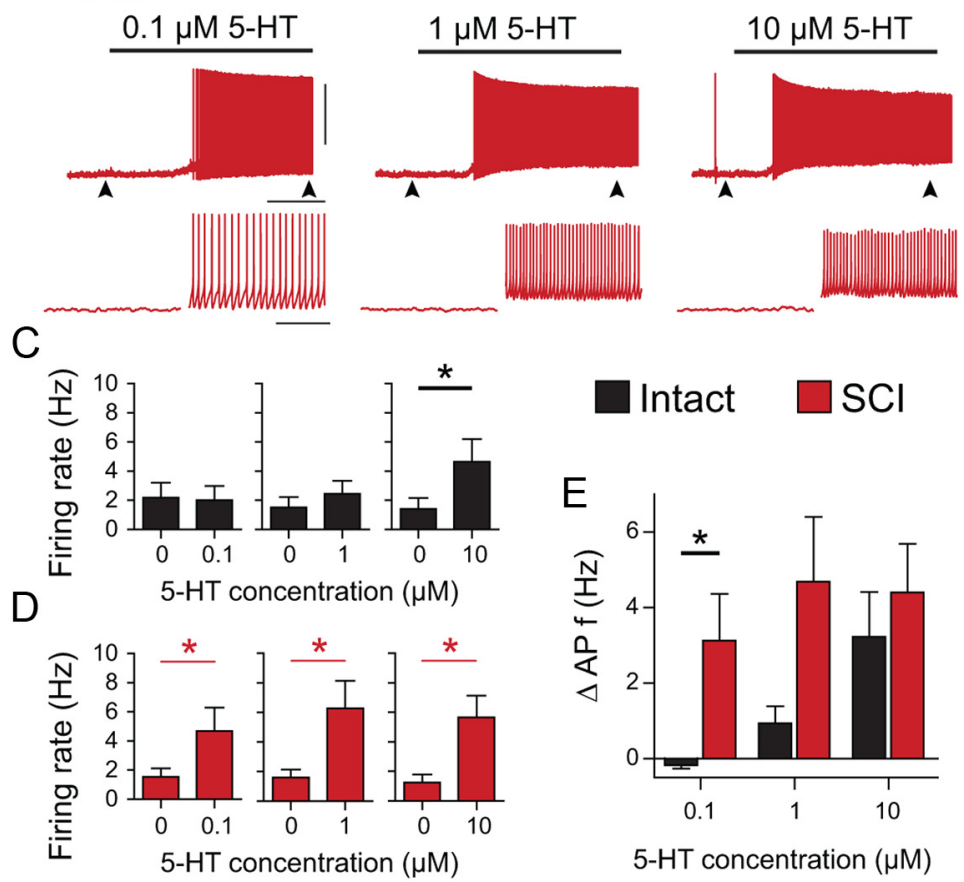

5-HT concentration $(\mu \mathrm{M})$

$\mathrm{F}$
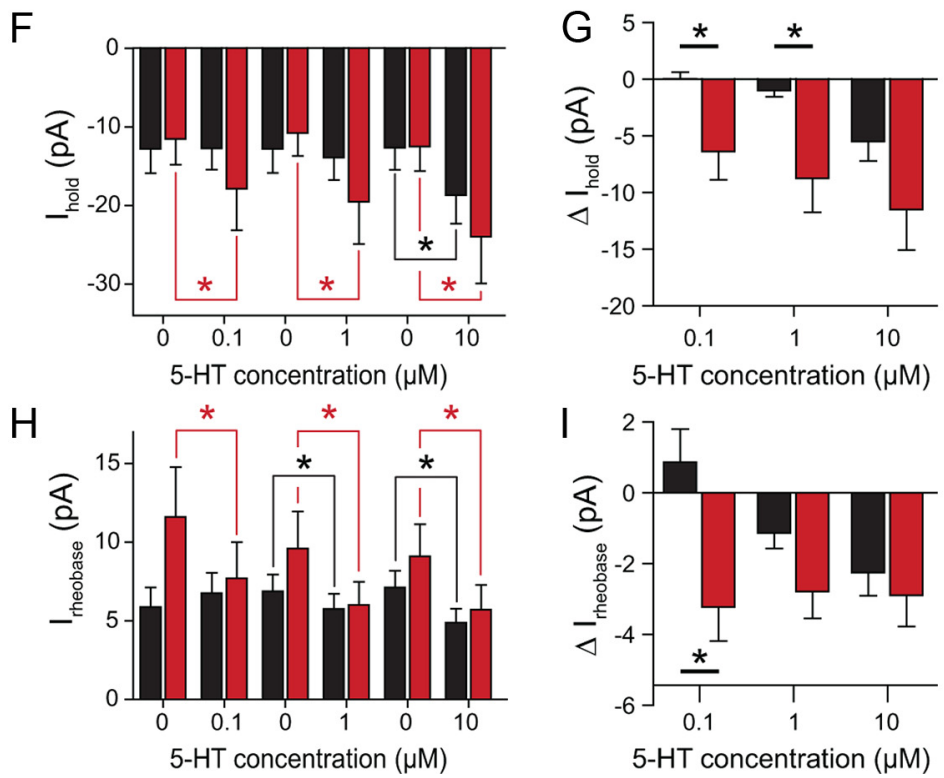

Figure 3. Enhanced serotonin (5-HT) sensitivity of V2a INs 4 weeks after SCl. $\boldsymbol{A}, \boldsymbol{B}$, Spontaneous firing activity of a typical intact neuron $(\boldsymbol{A})$ and SCIneuron $(\boldsymbol{B})$ during application of increasing 5-HT concentrations. Arrowheads indicate time of expanded time scale insets below. $\boldsymbol{C}, \boldsymbol{D}$, Average AP frequency of intact $(\boldsymbol{C})$ and SCI (D) neurons at different 5-HT concentration. $\boldsymbol{E}$, Change in AP frequency (intact: $n=7 ; \mathrm{SCl}$ : $n=8)$. $\boldsymbol{F}$, Average holding currents $\left(I_{\text {hold }}\right)$ at different $5-\mathrm{HT}$ concentration applications in intact and $\mathrm{SCl}$ cells. G, Change in holding current to hold the membrane potential at $E_{M}=-60 \mathrm{mV}$ (intact: $\left.n=10 ; \mathrm{SCl}: n=10\right)$. $\boldsymbol{H}$, Average rheobase values at different 5 -HT concentration applications in intactand SCI cells.I, Change in rheobase, the currentneeded to elicitan APat $E_{\mathrm{M}}=-60 \mathrm{mV}$ (intact: $\left.n=8 ; S C l: n=9\right)$. ${ }^{*} p<0.05$. 


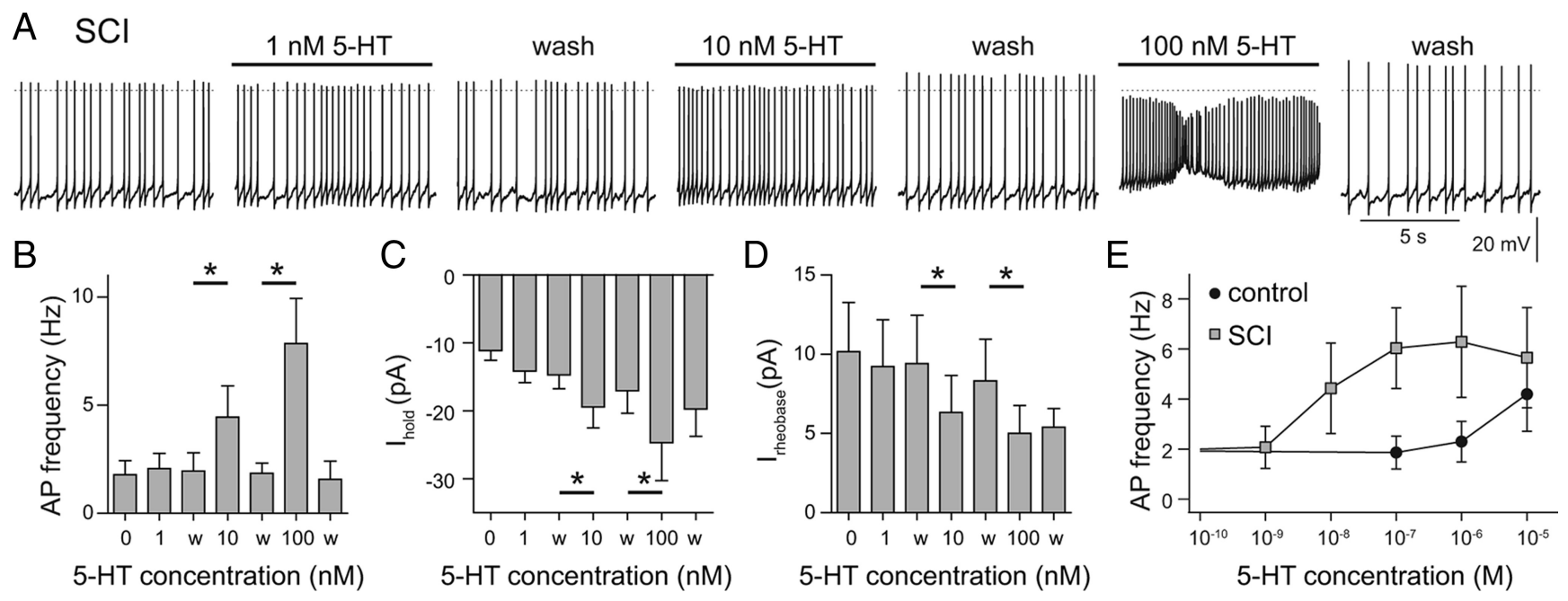

Figure 4. V2a INs show 100- to 1000-fold increase in 5-HT sensitivity after SCl. $\boldsymbol{A}$, Increase in spontaneous spike frequency during application of 1-100 nm 5-HT in an SCI V2a IN. $\boldsymbol{B}-\boldsymbol{D}$, Dose dependence of 5 -HT effects on average firing rate $(\boldsymbol{B})$, holding current at $-60 \mathrm{mV}\left(I_{\text {hold }}\right)(\boldsymbol{C})$, and rheobase $\left(I_{\text {rheobase }}\right)(\boldsymbol{D})$. Data in $\boldsymbol{B}-\boldsymbol{D}$ are means from six independent experiments in $\mathrm{SCl}$ mice. $\boldsymbol{E}$, Comparison of the 5-HT concentration dependence of the averaged, pooled AP firing rate for intact and SCI V2a neurons. $n=6-14$ for each data point. ${ }^{*} p<0.05$.

neurons were similar, $\sim 2 \mathrm{~Hz}$ (Fig. $1 C ; p=0.8$ ). Among the intact and SCI neurons that were silent or firing slowly $(<0.8 \mathrm{~Hz})$, the resting membrane potentials $\left(V_{m}\right)$ were similar, near $-52 \mathrm{mV}(p$ $=0.9 ; n=11-18 ;$ Fig. $1 D)$. We measured the intrinsic excitability of V2a INs by holding the neurons at $-60 \mathrm{mV}$, applying a series of depolarizing current steps and plotting the AP frequency as a function of current amplitude (Fig. $1 E, F$ ). Again, there was no sign of increased excitability 1 month after SCI $(p=0.3, n=17$ for both groups, regression analysis; Fig. $1 F)$. The capacitance of the somata was also unchanged ( $p=0.2, n=20-26)$. The only parameter to change significantly was the input resistance at -60 $\mathrm{mV}$, which was significantly lower in the SCI neurons (intact: $R_{\text {input }}=1.6 \pm 0.1 \mathrm{G} \Omega, n=29$; SCI: $R_{\text {input }}=1.1 \pm 0.1 \mathrm{G} \Omega, n=20$; $p=0.005$; Fig. $1 G, H)$. Consistent with this, the rheobase value, measured as the minimal current to evoke an AP from $-60 \mathrm{mV}$, showed a nonsignificant trend to be larger 1 month after SCI ( $p=$ $0.07, n=20-29$; Fig. $1 I$ ). We also examined the resting state by measuring the current $\left(I_{\text {hold }}\right)$ necessary to hold the membrane potential at $-60 \mathrm{mV}$; this holding current did not differ significantly between intact and SCI neurons $(p=0.4, n=20-29$; Fig. $1 J)$. We next analyzed the AP properties of V2a INs from intact and SCI mice. The membrane potential was held just below the neuron's spike threshold, so that low-frequency $(<1 \mathrm{~Hz})$ spontaneous APs could be recorded and signal averaged (Fig. $2 A$ ). The AP amplitude, threshold, half-width, the fast AHP amplitude (AHPf), and time to peak hyperpolarization (AHPf time), as well as the slow afterhyperpolarization amplitude (AHPs) and time (AHPs time), were not altered by SCI ( $p>0.05$ for all comparisons; Fig. $2 B-H)$. In summary, there is no evidence for increased excitability in V2a INs after SCI; in fact, the decreased input resistance and trend to increased rheobase indicate a trend toward a decreased excitability of V2a INs 4 weeks after SCI.

\section{SCI causes increased 5-HT sensitivity in V2a INs}

Several previous studies have shown that the spinal locomotor CPG (Shibuya and Anderson, 1968; Barbeau and Bédard, 1981), as well as sacral MNs (Harvey et al., 2006a) become supersensitive to serotonin (5-HT) after SCI. Thus, we compared the sensitivity of synaptically isolated V2a INs to serotonin in intact and SCI mice. We previously showed that a majority of neonatal mouse V2a INs are excited by 5 and $10 \mu \mathrm{M}$ serotonin (Zhong et al., 2010), so we tested adult V2a responses to $0.1,1$, and $10 \mu \mathrm{M} 5$-HT (Fig. 3 ). We recorded $\mathrm{V} 2 \mathrm{a}$ membrane properties under control conditions, during a $7 \mathrm{~min}$ perfusion of 5 -HT and during a wash period of 15 to $60 \mathrm{~min}$, which allowed for complete recovery to baseline before the next concentration of 5-HT was applied $(p>0.05$, repeated-measures ANOVA and post hoc tests for all control and recovery parameters). Intact V2a INs did not significantly increase their firing rate in response to $0.1 \mu \mathrm{M} 5-\mathrm{HT}(p=0.1)$ or 1 $\mu$ M 5-HT $(p=0.08)$. However, $80 \%(17 / 21)$ of intact V2a INs responded to $10 \mu \mathrm{M} 5$-HT with membrane potential depolarization, causing a significant increase in spontaneous firing rate from $1.4 \mathrm{~Hz}$ in control to $4.6 \mathrm{~Hz}$ during application $(p=0.04$, $n=7$; Fig. $3 A, C)$. In contrast, SCI V2a INs depolarized and significantly increased their firing rate from 1.5 to $4.7 \mathrm{~Hz}$ after application of $0.1 \mu \mathrm{M} 5$-HT $(p=0.04, n=8$; Fig. $3 B, D)$. After complete reversal on washout, application of $1 \mu \mathrm{M} 5$-HT accelerated the SCI neuron firing frequency significantly from 1.6 to $6.3 \mathrm{~Hz}(p=0.03)$. After up to $1 \mathrm{~h}$ wash to recover to baseline firing rates, a final addition of $10 \mu \mathrm{M} 5-\mathrm{HT}$ increased firing from 1.3 to $5.7 \mathrm{~Hz}(p=0.01$; Fig. $3 B, D)$. The increase in firing rate during $0.1 \mu \mathrm{M} 5$-HT was significantly higher in SCI than control neurons ( $p=0.03$; Fig. $3 E$ ). In SCI V2a INs, the maximal response was already achieved with $0.1 \mu \mathrm{M} 5-\mathrm{HT}$, and responses to higher 5 -HT concentrations were not significantly greater $(p=0.5$; Fig. $3 E)$.

We monitored 5-HT's depolarization of the V2a INs by measuring changes in the holding current $\left(I_{\text {hold }}\right)$ at $-60 \mathrm{mV}$. Serotonin did not change $I_{\text {hold }}$ in intact neurons at $0.1 \mu \mathrm{M}(p=0.9$, $n=10)$ or $1 \mu \mathrm{M} 5$-HT $(p=0.1)$, but significantly increased $I_{\text {hold }}$ from -12.7 to $-18.7 \mathrm{pA}$ during $10 \mu \mathrm{M} 5$-HT $(p=0.008$; Fig. $3 F)$. SCI V2a INs depolarized significantly and increased $I_{\text {hold }}$ with $0.1 \mu \mathrm{M}, 1 \mu \mathrm{M}$, and $10 \mu \mathrm{M}$ 5-HT $(p<0.03$ for all three concentrations, $n=10$; Fig. $3 F$ ); again, the effect was already maximal at $0.1 \mu \mathrm{M} 5$-HT (Fig. $3 G$ ). The increase in $I_{\text {hold }}$ during 5 -HT was greater in SCI than intact cells at 0.1 and $1 \mu \mathrm{M} 5$-HT $(p<0.02$ for both comparisons; Fig. $3 G$ ). Similar differences were seen with 5-HT modulation of the rheobase (Fig. $3 H$ ). Intact neurons decreased the rheobase only during 1 and $10 \mu \mathrm{M}$ 5-HT ( $p<0.04$ for both comparisons; $n=8$ ), while SCI V2a INs showed a significant reduction in rheobase at all three 5-HT con- 
centrations (Fig. $3 H$ ), with a larger effect at $0.1 \mu \mathrm{M} 5$-HT than control neurons $(p=0.008$; Fig. 3I).

As the effect of 5-HT was already maximal at $0.1 \mu \mathrm{M} 5$-HT in SCI V2a INs, we retested SCI V2a INs with 1,10 , and 100 nм 5-HT. Application of $1 \mathrm{~nm} 5$-HT had no effect $(p=0.3, n=6$; Fig. $4 A, B)$, but $10 \mathrm{~nm} 5$-HT $(p=0.02)$ and $100 \mathrm{~nm} 5$-HT $(p=0.02)$ significantly increased spontaneous SCI V2a IN firing rate (Fig. 4B). The holding current at $-60 \mathrm{mV}$ was not reversibly changed during $1 \mathrm{nM} 5$-HT, but 10 and 100 nM 5-HT both produced a robust and reversible increase in holding current ( $p<0.03$ in both cases; Fig. 4C). Changes in rheobase in SCI V2a neurons showed the same 5-HT sensitivity, with no change at $1 \mathrm{~nm} 5$-HT $(p=0.1)$, and significant decreases during 10 and 100 nм 5-HT $(p<0.04$; Fig. $4 D)$. Measurements of the change in firing frequency over the full range from $1 \mathrm{~nm}$ to $10 \mu \mathrm{M}$ 5-HT show that SCI V2a INs reach halfmaximal activation at $\sim 10 \mathrm{~nm} 5-\mathrm{HT}$, whereas intact V2a INs do not respond to nM concentrations of 5-HT at all (Fig. $4 E)$. Thus, SCI induces a $100-$ to $1000-$ fold increase in serotonin sensitivity in V2a INs.

Reduction in serotonin (5-HT) and serotonin transporters (SERT) after SCI The large majority of the serotonin in the spinal cord is released from termini of raphe neurons arising in the brainstem (Carlsson et al., 1964); this descending supply is lesioned by complete SCI (Hayashi et al., 2010). Loss of these descending serotonin terminals would also eliminate the majority of the serotonin transporters (SERT), which remove serotonin from the synaptic cleft. This loss of SERT would increase the effective concentration of applied serotonin, if remaining glial and other sources of SERT could not make up for the loss of 5-HT reuptake. We performed an immunocytochemical analysis of 5-HT and SERT in medial lamina VIII (Fig. 5A) of intact and SCI mice. In intact cords, serotonin immunofluorescence labeling was detected as fibrous staining in the axons of the descending raphe neurons (Fig. 5B; Carlsson et al., 1964). As expected (Carlsson et al., 1963), there was no fibrous immunostaining after SCI (Fig. 5C). Both the percentage of pixels expressing 5-HT immunoreactivity and the intensity of pixels were significantly lower in SCI mice compared with intact mice ( $85 \%$ reduction in pixel number after SCI, $p<0.05$, matched-pair test; $84.6 \%$ reduction in intensity of pixels
A
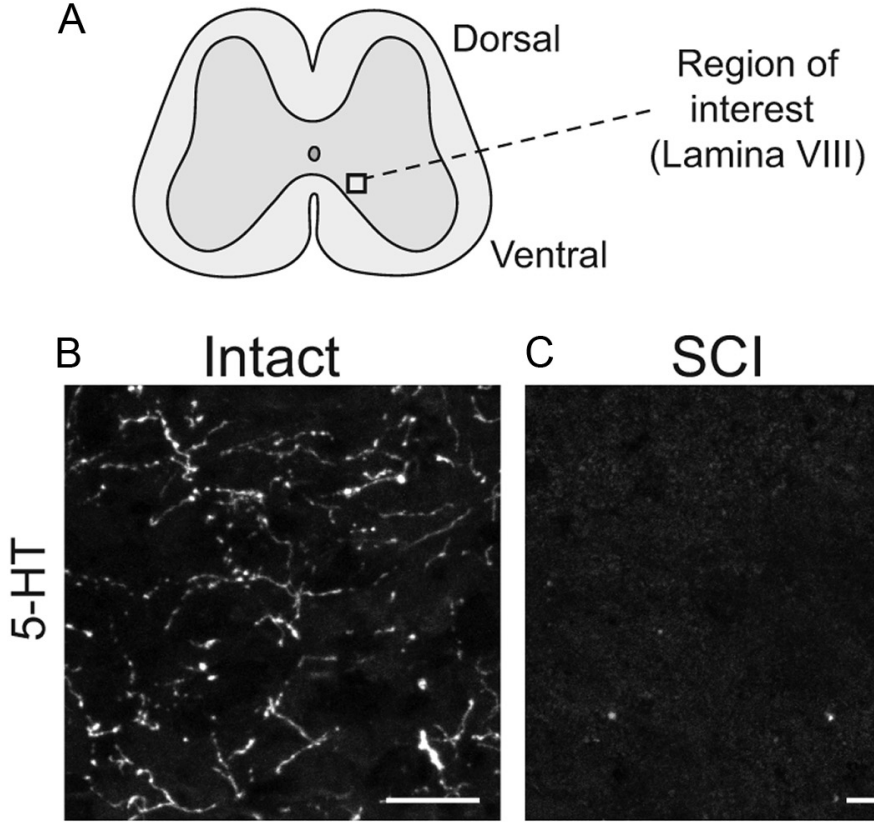

C $\mathrm{SCl}$
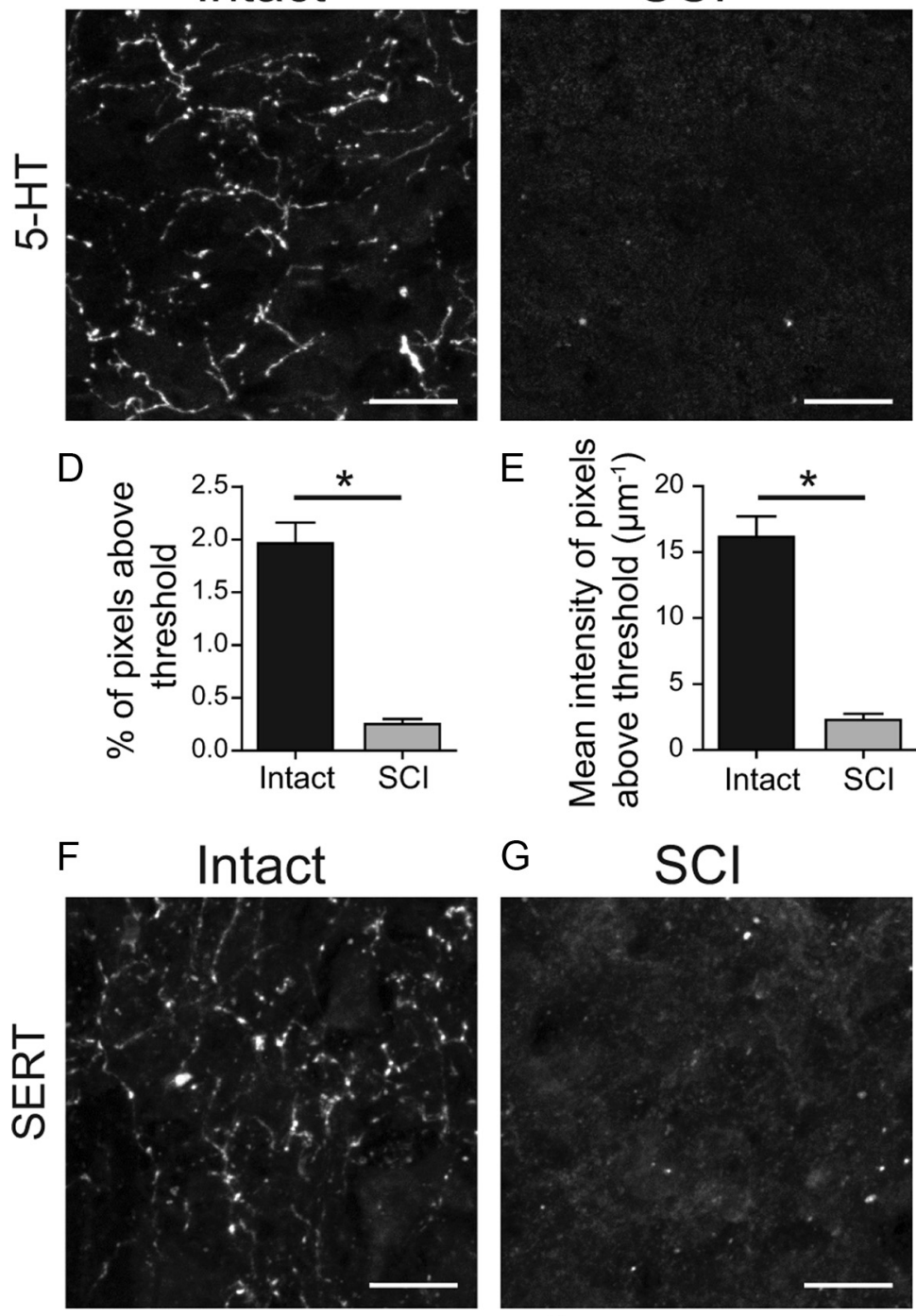

$\mathrm{G} \quad \mathrm{SCl}$

$\mathrm{H}$
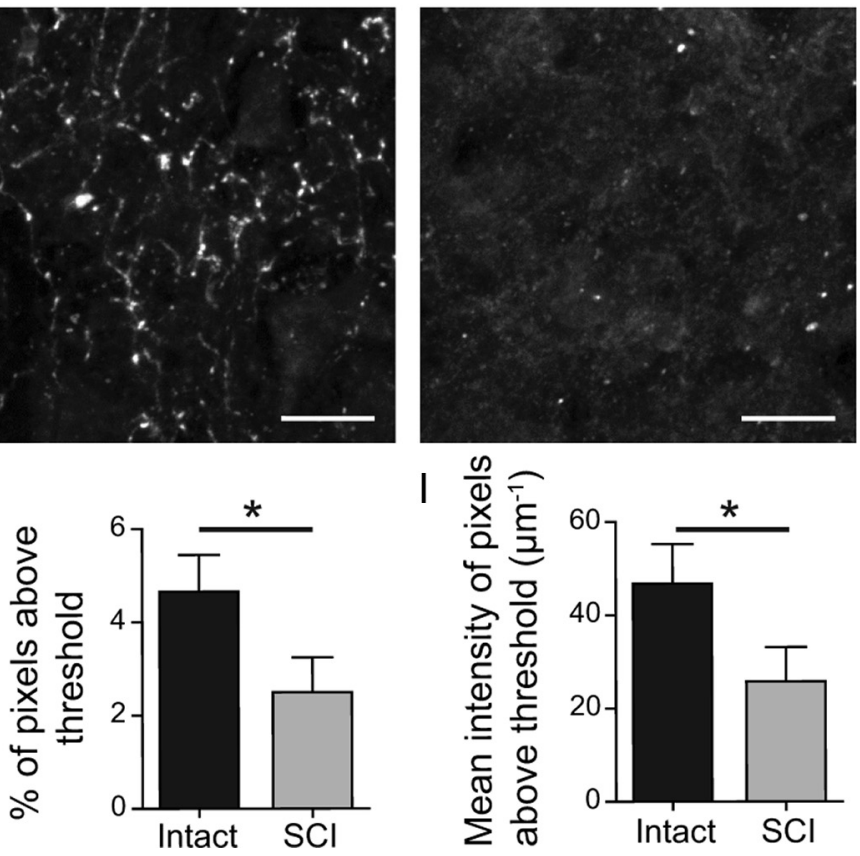

Figure 5. Reduction of serotonin (5-HT) and the 5 -HT transporter (SERT) immunoreactivity after SCI. $A$, Schematic spinal cord slice with region of interest located in the medial lamina VIII. $\boldsymbol{B}, \boldsymbol{C}, 5$-HT-immunoreactivity from intact $(\boldsymbol{B})$ and SCI mice $(\boldsymbol{C})$. D, E, Average area $(\boldsymbol{D})$ and intensity $(\boldsymbol{E})$ of 5-HT immunoreactivity in intact and SCl slices. $\boldsymbol{F}, \mathbf{G}$, SERT immunoreactivity from intact $(\boldsymbol{F})$ and SCl slices $(\boldsymbol{G}) . \boldsymbol{H}, \boldsymbol{I}, \mathrm{SCl}$ causes a significant decrease in the number of pixels above threshold $(\boldsymbol{H})$ and their mean intensity $(\boldsymbol{I})$ compared with slices from intactmice. ${ }^{*} p<$ 0.05 , matched pair test, $n=12$ pairs for 5 -HT and $n=6$ pairs for SERT. Scale bars: $10 \mu \mathrm{m}$. 


\section{A Intact}

\section{$10 \mu \mathrm{M}$ citalopram}
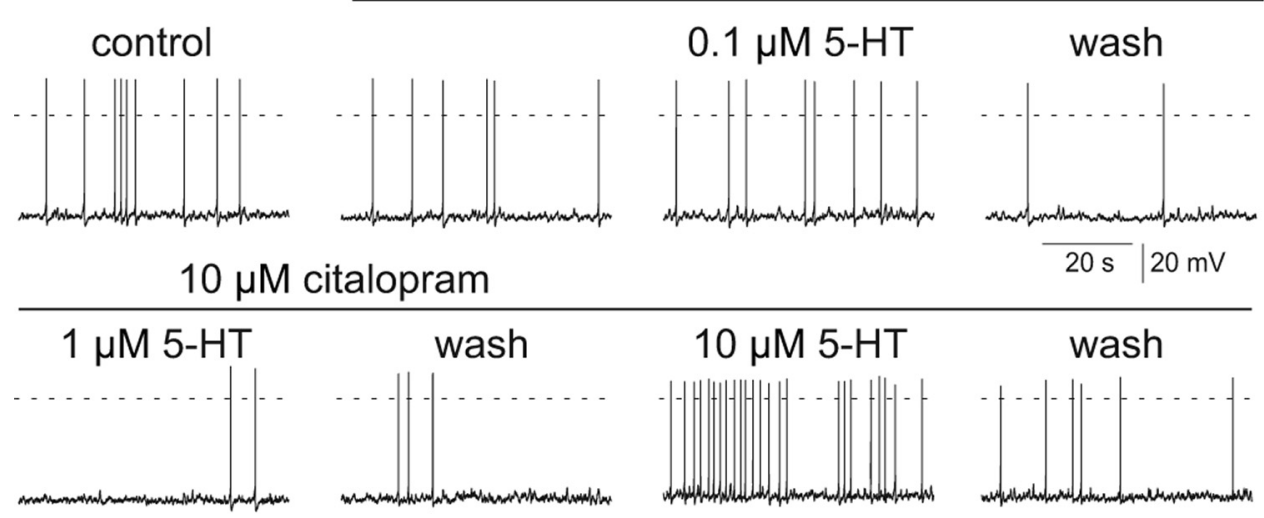

B

C
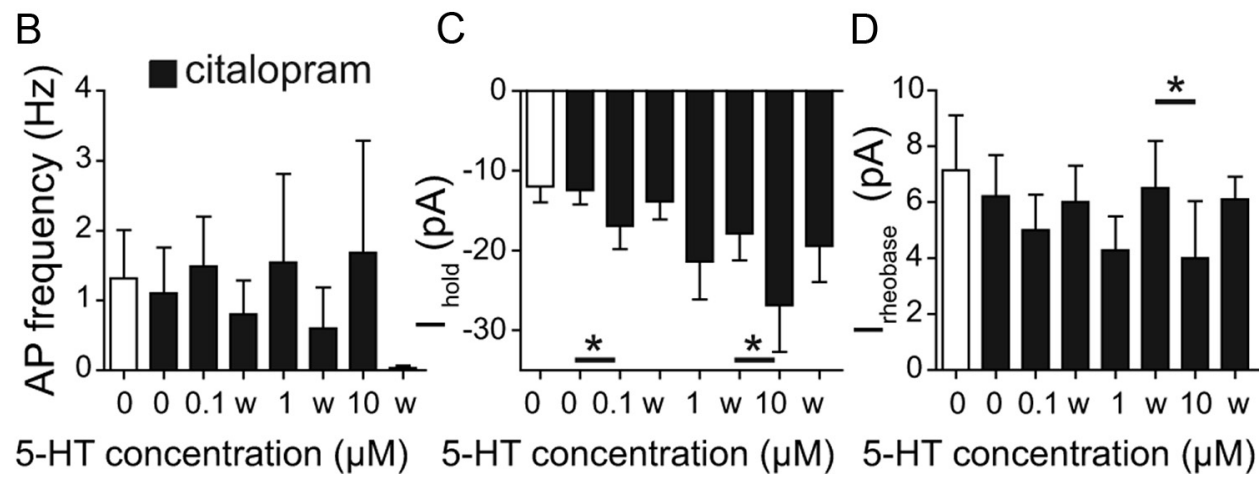

Figure 6. Serotonin transporter block in control slices does not mimic 5-HT sensitivity increase seen after SCl. $A$, Effect of citalopram (10 $\mu \mathrm{M}$ ) on spontaneous firing responses to $0.1,1$, and $10 \mu \mathrm{M}$ 5-HT of a V2a IN from an intact spinal cord slice. $B$, Average spontaneous V2a firing rate at different 5-HT concentrations of intact V2a INs pretreated with citalopram. C, Citalopram increases serotonin sensitivity of the holding current at $-60 \mathrm{mV}\left(I_{\text {hold }}\right)$. $\boldsymbol{D}$, Citalopram pretreatment does not alter the concentration dependence of the increase in rheobase in intact V2a INs. ${ }^{*} p<0.05$, data in $\boldsymbol{B}-\boldsymbol{D}$ are means from seven independent experiments in intact mice.

above threshold, $p<0.05$, matched-pair test; $n=12$; Fig. $5 D, E)$. As expected (Hayashi et al., 2010), fibrous SERT immunostaining was also seen in the ventromedial cord of intact mice (Fig. $5 F$ ), and this was greatly reduced after SCI (Fig. $5 G$ ). Both the percentage of SERT-labeled pixels and their intensity were significantly lower in SCI mice compared with intact mice (51.4\% decrease of total pixels after SCI, $p<0.05$, matched-pair test; $49.4 \%$ decrease in remaining pixel intensity after SCI, $p<0.05 ; n=6$, Fig. $5 H, I)$. Presumably this reflects the loss of SERT on 5-HT terminals after SCI. Pre-absorption of the SERT antibody with the SERT peptide epitope used to raise the antibody eliminated virtually all labeling.

\section{Influence of SERT block on 5-HT sensitivity in intact cord preparations}

To determine whether the loss of SERT on the lesioned serotonergic terminals could explain the SCI-induced increase in 5-HT sensitivity, we tested the effect of pharmacologically blocking SERT on 5-HT sensitivity of intact mice. Application of the SERT inhibitor citalopram (10 $\mu \mathrm{M}$; Murray et al., 2010) did not change spontaneous firing, $I_{\text {hold }}$ or the rheobase values of V2a neurons (Fig. 6). The average firing rate of serotonin-sensitive control neurons treated with citalopram did not increase significantly with $0.1 \mu \mathrm{M} 5$-HT $(p=0.3, n=7$; Fig. $6 A, B)$. During subsequent 1 and $10 \mu \mathrm{M} 5$-HT applications, only two of the seven cells increased firing. Though there was variability between neurons, none of the 5-HT concentrations significantly increased intact V2a firing rates in the presence of citalopram. However, the 5-HT effects on the holding current suggest that the neurons in intact mice became slightly more sensitive to 5-HT after SERT blockade. All 5-HT-sensitive cells (7/10) responded to $0.1 \mu \mathrm{M} 5-\mathrm{HT}$ with weak depolarization, resulting in a significantly increased $I_{\text {hold }}(p=0.02, n=7$; Fig. $6 C)$. Application of $1 \mu \mathrm{M} 5$-HT in citalopram had a similar effect, but it was not quite significant $(p=0.054)$, and did not wash out completely after removal of 5-HT. This may reflect the important role of the 5-HT transporter for complete washout and recovery after addition of higher concentrations of 5-HT. Application of $10 \mu \mathrm{M} 5$-HT significantly increased $I_{\text {hold }}(p=0.02$; Fig. $6 C$ ). Serotonin slightly decreased the mean rheobase value at lower concentrations, but this effect was only statistically significant at $10 \mu \mathrm{M} 5-\mathrm{HT}$ ( $p=$ 0.003; Fig. $6 D$ ). In summary, blocking the serotonin transporter in intact V2a INs causes a weak and variable increase in 5-HT sensitivity that is perhaps complicated by the effects of $5-\mathrm{HT}$ on inhibitory as well as excitatory receptors (see Discussion). Comparing the effects of $0.1 \mu \mathrm{M} \mathrm{5-HT}$ in the intact + citalopram neurons to the SCI neurons, it is clear that the loss of SERT cannot completely explain the SCI-induced increase in 5-HT sensitivity.

\section{Increase in $5-\mathrm{HT}_{2 \mathrm{C}}$ receptors after $\mathrm{SCI}$}

An alternative explanation for the increased 5-HT sensitivity after SCI is an upregulation of 5-HT receptors on V2a INs. As a first step to testing this, we examined the expression of $5-\mathrm{HT}_{2 \mathrm{C}}$ receptors, one of the main excitatory 5-HT receptors in the spinal cord (Schmidt and Jordan, 2000), in medial lamina VIII in control and 
SCI mice (Fig. $7 A, B$ ). Using a $5-\mathrm{HT}_{2 \mathrm{C}}$ antibody, $5-\mathrm{HT}_{2 \mathrm{C}}$ expression in control cells was punctate, presumably reflecting the clustering of receptors at serotonergic synapses. The number of $5-\mathrm{HT}_{2 \mathrm{C}}$ receptor clusters was significantly greater in SCI mice compared with intact mice $(74 \%$ increase, $p<0.01, n=13$; Fig. $7 C$ ). The mean area of the $5-\mathrm{HT}_{2 \mathrm{C}}$ clusters was also significantly greater after SCI $(92 \%$ increase; $p<0.01$; Fig. $7 D$ ). Finally, the intensity of cluster labeling, presumably reflecting the density of $5-\mathrm{HT}_{2 \mathrm{C}}$ receptors within the cluster, was not significantly different in control and SCI cords $(p>$ 0.05 ; Fig. 7E). Pre-absorption of the $5-\mathrm{HT}_{2 \mathrm{C}}$ antibody with the $5-\mathrm{HT}_{2 \mathrm{C}}$ peptide epitope used to raise the antibody eliminated all labeling. These results suggest that $\mathrm{SCI}$ increases $5-\mathrm{HT}_{2 \mathrm{C}}$ expression in V2a INs.

\section{Discussion}

While most SCI research focuses on repairing the primary lesion and stimulating descending axon regrowth, regulating the compensatory changes in sublesional neurons and networks may also be very important. The cellular mechanisms underlying plastic changes after SCI have only been studied in detail in MNs. Immediately after SCI, with the accompanying loss of 5-HT, MNs become less excitable (Hounsgaard et al., 1988). However, several weeks after SCI, rat sacral MNs become hyperexcitable (Harvey et al., 2006b). Their input resistance increases significantly, and the MNs develop chronic unregulated bistability (Bennett et al., 2001a; Li and Bennett, 2003). The depolarized state is supported by an SCI-induced upregulation of both sodium and calcium PICs (Li and Bennett, 2003; Li et al., 2004, 2007; Harvey et al., 2006b), mediated in part by an upregulation of $\mathrm{Ca}_{\mathrm{V}} 1.2$ calcium channels (Anelli et al., 2007). In control spinal cords, these PICs are dependent on serotonergic and noradrenergic activation, and are reduced by $5-\mathrm{HT}_{2}$ and $\alpha_{1}$ antagonists in both control and SCI MNs. After SCI, altered RNA editing increases constitutive activity in $5-\mathrm{HT}_{2 \mathrm{C}} \mathrm{re}-$ ceptors, which supports MN hyperexcitability (Murray et al., 2010). This higher excitability can cause long-lasting reflex responses and spasticity. Blocking $5-\mathrm{HT}_{2 \mathrm{C}}$ constitutive activity decreases spasticity after SCI in rats and humans (Murray et al., 2010). In contrast to this extensive work on MNs, changes in intrinsic excitability of spinal locomotor-related INs have not been previously examined. Based on the earlier MN results, it was expected that INs would also show hyperexcitability (Elbasiouny et al., 2010). However, in our study, we found no sign of increased excitability in V2a INs 1 month after SCI. To the contrary, we found the membrane input resistance to be significantly decreased; this shifted the rheobase to higher values, indicating a trend to even lower excitability than intact cells. Spinal cord slices of uninjured mice contain serotonergic terminals, which could still release 5-HT during the experiment. Thus, residual 5-HT release might explain the higher input resistance of V2a INs in intact slices. However, other properties of SCI INs, including their AP properties, threshold, and excitability during current steps, were virtually identical in control and SCI neurons; there were no signs of increased bistability in SCI V2a INs. These results are in marked contrast to the effects of SCI on rat sacral MNs (Harvey et al., 2006b) where a reduced spike threshold contributed to the increased excitability after the injury. Our findings provide evidence for heterogeneity and cell type-specific responses to SCI. In addition, they could reflect either species or spinal cord level-specific differences in neuronal responses to SCI.

The most striking change in V2a INs after SCI was a 100- to 1000-fold increase in 5-HT sensitivity. Denervation supersensitivity to 5-HT has been shown to increase electromyographic activity in rat hindlimbs (Barbeau and Bédard, 1981), as well as enhancement of long-lasting reflexes (Li et al., 2004). On a cellular level, rat sacral MNs show a 30-fold increase in 5-HT sensitivity for facilitation of $\mathrm{Na}^{+}$PICs (Harvey et al., 2006a) and $\mathrm{Ca}^{2+}$ PICs (Li et al., 2007). This is the first demonstration of a dramatic increase in 5-HT sensitivity in an identified spinal IN type after SCI. V2a INs are glutamatergic and excitatory to commissural INs and MNs (Crone et al., 2008). Thus, the presence of minimal amounts of residual 5-HT in the cord after SCI, which has been measured in the rat spinal cord (Harvey et al., 2006c), could contribute to spasticity and hyper-reflexia. What are the mechanisms responsible for this remarkable increase of 5-HT sensitivity? In rats, SCI causes a marked decrease in 5-HT levels below the lesion (Hadjiconstantinou et al., 1984; Hayashi et al., 2010; Murray et al., 2010; Kong et al., 2011). We confirmed this finding in mice with an $85 \%$ reduction of 5-HT immunoreactivity. In rats, Hayashi et al. (2010) and Kong et al. (2011) found an almost complete loss of SERT with time after SCI. We also found a significant, though not complete, loss of SERT at 1 month post-SCI. The decrease in SERT immunoreactivity after SCI can be partially attributed to a degradation of serotonergic terminals, since 5-HT transporters are predominantly located at the synaptic 
bouton, and all fibrous SERT immunolabeling was lost after SCI (Fig. 5). The SERT labeling remaining after SCI could result from weak nonspecific labeling by the SERT antibody, or SERT expression by other neurons or glial cells (Inazu et al., 2001 ) in the medial lamina VIII region. Species differences could also contribute to the relatively smaller elimination of SERT and 5-HT after SCI in our experiments with mice, compared with previous work using rats. The weak impact of blocking SERT activity on V2a neuron 5-HT responses in intact mice indicated that the reduction of SERT is not the primary cause for increased 5-HT sensitivity after SCI. Application of citalopram did not shift the 5-HT sensitivity to nanomolar concentrations. Variability of the excitatory 5-HT effects during citalopram might be caused by a shift in the ratio of opposing 5-HT receptor responses, e.g., excitatory $5-\mathrm{HT}_{2 \mathrm{C}}$ and inhibitory $5-\mathrm{HT}_{1 \mathrm{~A}}$. receptors. Sensitivity characterization with specific 5-HT receptor agonists will be necessary to fully understand the exact contribution of the loss of SERT in increased V2a IN sensitivity to 5-HT, but by itself, this loss does not explain the increased sensitivity.

After SCI, we also found a significant upregulation in the number and size of $5-\mathrm{HT}_{2 \mathrm{C}}$ receptor clusters. Because $5-\mathrm{HT}_{2 \mathrm{C}}$ receptors have postsynaptic functions in lumbar medial lamina VIII, we would expect such a homeostatic upregulation in $5-\mathrm{HT}_{2 \mathrm{C}}$ levels after SCI. The increase in number of $5-\mathrm{HT}_{2 \mathrm{C}}$ receptor clusters may represent new sites of $5-\mathrm{HT}_{2 \mathrm{C}}$ expression on dendrites. The increase in average area of individual clusters after SCI could reflect an increased expression of $5-\mathrm{HT}_{2 \mathrm{C}}$ receptors at each cluster. However, the pixel intensity does not increase in each cluster, suggesting that after SCI, each $5-\mathrm{HT}_{2 \mathrm{C}}$ cluster covers more area than in control cords, but with no increase in density of $5-\mathrm{HT}_{2 \mathrm{C}}$ receptors.

In conclusion, V2a INs, which are components of the locomotor CPG network, respond remarkably differently to SCI then the well studied MNs. They do not show an increased intrinsic excitability, but do show a very marked supersensitivity to 5-HT. There appear to be many causes for this supersensitivity, including lesion-induced reductions in SERT and increased expression of $5-\mathrm{HT}_{2 \mathrm{C}}$ receptors that mediate depolarizing 5-HT responses. It is likely that other receptor subtypes and channels are also affected in different ways by SCI. Other IN types (such as V0, V1, $\mathrm{V} 2, \mathrm{~V} 3$, and HB9 INs) may react to SCI in very different ways from the V2a INs, especially because V2a INs are preferentially recruited during high locomotor frequencies compared with other IN classes that are evoked at normal locomotor frequencies. It is important to understand the possibly different remodeling of all components in the network to design effective therapies to restore function following SCI and minimize the potential for exacerbating pathological conditions such as spasticity or neuropathic pain. Using multiple approaches such as immunohistochemistry and electrophysiology can elucidate the multiple neuronal responses to SCI, and how we can adjust those effects to increase the quality of life after SCI.

\section{References}

Anelli R, Sanelli L, Bennett DJ, Heckman CJ (2007) Expression of L-type calcium channel $\alpha 1-1.2$ and $\alpha 1-1.3$ subunits on rat sacral motoneurons following chronic spinal cord injury. Neuroscience 145:751-763.

Antri M, Mouffle C, Orsal D, Barthe JY (2003) 5-HT1A receptors are involved in short- and long-term processes responsible for 5-HT-induced locomotor function recovery in chronic spinal rat. Eur J Neurosci 18:1963-1972.

Antri M, Barthe JY, Mouffle C, Orsal D (2005) Long-lasting recovery of locomotor function in chronic spinal rat following chronic combined pharmacological stimulation of serotonergic receptors with 8-OHDPAT and quipazine. Neurosci Lett 384:162-167.

Barbeau H, Bédard P (1981) Denervation supersensitivity to 5-hydroxytryptophan in rats following spinal transection and 5,7-dihydroxytryptamine injection. Neuropharmacology 20:611-616.

Bennett DJ, Li Y, Siu M (2001a) Plateau potentials in sacrocaudal motoneurons of chronic spinal rats, recorded in vitro. J Neurophysiol 86:1955-1971.

Bennett DJ, Li Y, Harvey PJ, Gorassini M (2001b) Evidence for plateau potentials in tail motoneurons of awake chronic spinal rats with spasticity. J Neurophysiol 86:1972-1982.

Berg KA, Cropper JD, Niswender CM, Sanders-Bush E, Emeson RB, Clarke WP (2001) RNA-editing of the 5-HT(2C) receptor alters agonistreceptor-effector coupling specificity. Br J Pharmacol 134:386-392.

Carlsson A, Magnusson T, Rosengren E (1963) 5-hydroxytryptamine of the spinal cord normally and after transection. Experientia 19:359.

Carlsson A, Falck B, Fuxe K, Hillarp NA (1964) Cellular localization of monoamines in the spinal cord. Acta Physiol Scand 60:112-119.

Crone SA, Quinlan KA, Zagoraiou L, Droho S, Restrepo CE, Lundfald L, Endo T, Setlak J, Jessell TM, Kiehn O, Sharma K (2008) Genetic ablation of V2a ipsilateral interneurons disrupts left-right locomotor coordination in mammalian spinal cord. Neuron 60:70-83.

Dougherty KJ, Kiehn O (2010) Firing and cellular properties of V2a interneurons in the rodent spinal cord. J Neurosci 30:24-37.

Edgerton VR, Tillakaratne NJ, Bigbee AJ, de Leon RD, Roy RR (2004) Plasticity of the spinal neural circuitry after injury. Annu Rev Neurosci 27:145-167.

Elbasiouny SM, Moroz D, Bakr MM, Mushahwar VK (2010) Management of spasticity after spinal cord injury: current techniques and future directions. Neurorehabil Neural Repair 24:23-33.

Hadjiconstantinou M, Panula P, Lackovic Z, Neff NH (1984) Spinal cord serotonin: a biochemical and immunohistochemical study following transection. Brain Res 322:245-254.

Harvey PJ, Li X, Li Y, Bennett DJ (2006a) 5-HT2 receptor activation facilitates a persistent sodium current and repetitive firing in spinal motoneurons of rats with and without chronic spinal cord injury. J Neurophysiol 96:1158-1170.

Harvey PJ, Li Y, Li X, Bennett DJ (2006b) Persistent sodium currents and repetitive firing in motoneurons of the sacrocaudal spinal cord of adult rats. J Neurophysiol 96:1141-1157.

Harvey PJ, Li X, Li Y, Bennett DJ (2006c) Endogenous monoamine receptor activation is essential for enabling persistent sodium currents and repetitive firing in rat spinal motoneurons. J Neurophysiol 96:1171-1186.

Hayashi Y, Jacob-Vadakot S, Dugan EA, McBride S, Olexa R, Simansky K, Murray M, Shumsky JS (2010) 5-HT precursor loading, but not 5-HT receptor agonists, increases motor function after spinal cord contusion in adult rats. Exp Neurol 221:68-78.

Hounsgaard J, Hultborn H, Jespersen B, Kiehn O (1988) Bistability of alpha-motoneurones in the decerebrate cat and in the acute spinal cat after intravenous 5-hydroxytryptophan. J Physiol 405:345-367.

Husch A, Cramer N, Harris-Warrick RM (2011) Long-duration perforated patch recordings from spinal interneurons of adult mice. J Neurophysiol 106:2783-2789.

Hutcheon B, Brown LA, Poulter MO (2000) Digital analysis of light microscope immunofluorescence: high-resolution co-localization of synaptic proteins in cultured neurons. J Neurosci Methods 96:1-9.

Inazu M, Takeda H, Ikoshi H, Sugisawa M, Uchida Y, Matsumiya T (2001) Pharmacological characterization and visualization of the glial serotonin transporter. Neurochem Int 39:39-49.

Kong XY, Wienecke J, Chen M, Hultborn H, Zhang M (2011) The time course of serotonin $2 \mathrm{~A}$ receptor expression after spinal transection of rats: an immunohistochemical study. Neuroscience 177:114-126.

Lapointe NP, Guertin PA (2008) Synergistic effects of D1/5 and 5-HT1A/7 receptor agonists on locomotor movement induction in complete spinal cord-transected mice. J Neurophysiol 100:160-168.

Li X, Murray K, Harvey PJ, Ballou EW, Bennett DJ (2007) Serotonin facilitates a persistent calcium current in motoneurons of rats with and without chronic spinal cord injury. J Neurophysiol 97:1236-1246.

Li Y, Bennett DJ (2003) Persistent sodium and calcium currents cause plateau potentials in motoneurons of chronic spinal rats. J Neurophysiol 90:857-869. 
Li Y, Harvey PJ, Li X, Bennett DJ (2004) Spastic long-lasting reflexes of the chronic spinal rat studied in vitro. J Neurophysiol 91:22362246.

Murray KC, Nakae A, Stephens MJ, Rank M, D’Amico J, Harvey PJ, Li X, Harris RL, Ballou EW, Anelli R, Heckman CJ, Mashimo T, Vavrek R, Sanelli L, Gorassini MA, Bennett DJ, Fouad K (2010) Recovery of motoneuron and locomotor function after spinal cord injury depends on constitutive activity in 5-HT2C receptors. Nat Med 16: 694-700.

Ribotta MG, Provencher J, Feraboli-Lohnherr D, Rossignol S, Privat A, Orsal D (2000) Activation of locomotion in adult chronic spinal rats is achieved by transplantation of embryonic raphe cells reinnervating a precise lumbar level. J Neurosci 20:5144-5152.

Rossignol S (2000) Locomotion and its recovery after spinal injury. Curr Opin Neurobiol 10:708-716.

Schmidt BJ, Jordan LM (2000) The role of serotonin in reflex modulation and locomotor rhythm production in the mammalian spinal cord. Brain Res Bull 53:689-710.

Shibuya T, Anderson EG (1968) The influence of chronic cord transection on the effects of 5-hydroxytryptophan, 1-tryptophan and pargyline on spinal neuronal activity. J Pharmacol Exp Ther 164:185-190.

van den Brand R, Heutschi J, Barraud Q, DiGiovanna J, Bartholdi K, Huerlimann M, Friedli L, Vollenweider I, Moraud EM, Duis S, Dominici N, Micera S, Musienko P, Courtine G (2012) Restoring voluntary control of locomotion after paralyzing spinal cord injury. Science 336:1182-1185.

Zhong G, Droho S, Crone SA, Dietz S, Kwan AC, Webb WW, Sharma K, Harris-Warrick RM (2010) Electrophysiological characterization of V2a interneurons and their locomotor-related activity in the neonatal mouse spinal cord. J Neurosci 30:170-182.

Zhong G, Sharma K, Harris-Warrick RM (2011) Frequency-dependent recruitment of V2a interneurons during fictive locomotion in the mouse spinal cord. Nat Commun 2:274. 